Natural Laws and the Problem of Provisos

Author(s): Marc Lange

Source: Erkenntnis (1975-), Vol. 38, No. 2, Special Issue on Philosophy of Science (Mar., 1993), pp. 233-248

Published by: Springer

Stable URL: http://www.jstor.org/stable/20012470

Accessed: 12/11/2013 21:02

Your use of the JSTOR archive indicates your acceptance of the Terms \& Conditions of Use, available at http://www.jstor.org/page/info/about/policies/terms.jsp

JSTOR is a not-for-profit service that helps scholars, researchers, and students discover, use, and build upon a wide range of content in a trusted digital archive. We use information technology and tools to increase productivity and facilitate new forms of scholarship. For more information about JSTOR, please contact support@jstor.org. 


\title{
NATURAL LAWS AND THE PROBLEM OF PROVISOS
}

\begin{abstract}
Hempel and Giere contend that the existence of provisos poses grave difficulties for any regularity account of physical law. However, Hempel and Giere rely upon a mistaken conception of the way in which statements acquire their content. By correcting this mistake, I remove the problem Hempel and Giere identify but reveal a different problem that provisos pose for a regularity account - indeed, for any account of physical law according to which the state of affairs described by a law-statement presupposes a Humean regularity. These considerations suggest a normative analysis of law-statements. On this view, law-statements are not distinguished from accidental generalizations by the kind of Humean regularities they describe because a law-statement need not describe any Humean regularity. Rather, a law-statement says that in certain contexts, one ought to regard the assertion of a given type of claim, if made with justification, as a proper way to justify a claim of a certain other kind.
\end{abstract}

According to the regularity account of physical law - versions of which have been advocated by Ayer (1963), Braithwaite (1953, ch. 9), Goodman (1983, pp. 17-27), Hempel (1965a, pp. 264ff.), Lewis (1973, pp. 72-77; 1986), Mackie (1962, pp. 71-73), Nagel (1961, pp. 58ff.), and Reichenbach (1947, ch. 8), among others - laws of nature are regularities among events or states of affairs and a law-statement, the linguistic expression of a law, is a description of a regularity that is a law. The familiar challenge faced by this account is to distinguish those descriptions of regularities that are law-statements from those that are accidental generalizations. I wish to consider a more fundamental problem: that many a claim we believe to describe no regularity at all, nomological or accidental, we nevertheless accept as a law-statement. This problem arises from what Hempel (1988) calls "the problem of provisos."

Consider the familiar statement of the law of thermal expansion: "Whenever the temperature of a metal bar of length $L_{0}$ changes by $\Delta T$, the bar's length changes by $\Delta L=k \cdot L_{0} \cdot \Delta T$, where $k$ is a constant characteristic of that metal." This statement states a relation between $L_{0}, \Delta T$, and $\Delta L$ that does not obtain; it may be violated, for instance, if someone is hammering the rod inward at one end. Since this state-

Erkenntnis 38: 233-248, 1993.

(C) 1993 Kluwer Academic Publishers. Printed in the Netherlands. 
ment does not describe a regularity, it is not a law-statement, on the regularity account.

Hempel (1988) would hold that a complete statement of the law includes the condition ". . . if the end of the rod is not being hammered in." On this view, whereas the familiar "law-statement" takes the form (x) $(F x \supset G x)$, and the only premise one must add to yield the conclusion $G a$ is $F a$, the genuine law-statement takes the form $(x)$ ( $F x \& P x \supset G x$ ), and one needs to add $P a$ as well as $F a$ to infer $G a$. It is the regularity account of laws that leads Hempel to characterize such conditions $(x) \ldots$ if $P x$ as "essential" to law-statements (and the corresponding premises $P a$ as "essential" to inferences); without these conditions, the claims would be false and so, on the regularity account, would not be law-statements. Following Hempel (1988, p. 23), I'll refer to those conditions (and premises) that, by this reasoning, are necessary to law-statements (and to inferences from law-statements), but are "generally unstated," as "provisos."

Provisos pervade scientific practice. By Hempel's reasoning, Snell's law of refraction - when a beam of light passes from one medium to another, $\sin i / \sin r=$ constant, where $i$ is the angle of incidence of the beam upon the second medium, $r$ is the angle of refraction in that medium, and the constant is characteristic of the two types of media must require particular temperatures and pressures of the media as well as the absence of any magnetic or electrical potential difference across the boundary, uniform optical density and transparency and non-double-refractivity in the two media, and a monochromatic beam; these conditions are provisos. Likewise, the law of freely falling bodies - the distance a body falls to earth in time $t$ is $(1 / 2) g t^{2}-$ must specify when fall qualifies as "free"; while the law can remain approximately true away from the height at which $g$ is measured, its predictions may be drastically wrong when electromagnetic forces, air resistance, or other collisions affect the falling body.

On Hempel's proposal, it becomes impossible to state very many genuine law-statements since, as Giere puts it (1988, p. 40), "the number of provisos implicit in any law is indefinitely large." To state the law of thermal expansion, for instance, one would need to specify not only that no one is hammering the bar inward at one end, but also that the bar is not encased on four of its six sides in a rigid material that will not yield as the bar is heated, and so on. For that matter, not all cases in which the bar is hammered upon constitute exceptions to 
$\Delta L=k \cdot L_{0} \cdot \Delta T$; the bar may be hammered upon so softly and be on such a frictionless surface that the hammering produces translation rather than compression of the bar. One is driven to say that the only way to utter a complete law-statement is to employ some such condition as "... in the absence of other relevant factors." But Hempel deems such an expression inadmissible in a law-statement: On the regularity account, a law-statement states a particular relation (which must obtain and be a law), but a claim $(x)(F x \&$ there are no other relevant factors $\supset G x$ ) does not assert any determinate relation at all because it fails to specify which other factors count as relevant, i.e., which specific premises are to be added to $F a$ and the law-statement to infer $G a$. Such a claim is no better than "The relation $\Delta L=k \cdot L_{0} \cdot \Delta T$ holds when it holds," and so is further from being a law-statement than is the familiar statement of the law of thermal expansion, which at least ascribes a particular (albeit false) relation to various quantities.

In short, Hempel sees the existence of provisos as posing a dilemma: For many a claim that we commonly accept as a law-statement, either that claim states a relation that does not obtain, and so is false, or is shorthand for some claim that states no relation at all, and so is empty. In either case, the regularity account must admit that many claims commonly accepted as law-statements are neither complete law-statements themselves nor even colloquial stand-ins for complete law-statements. If we continue to regard those familiar claims as law-statements, then we violate the regularity account. This is the problem of provisos. (Prior to Hempel (1988), versions of this problem were discussed by Canfield and Lehrer (1961) and Coffa (1968), as well as by Hempel himself (1965b, p. 167), and the issue, in general terms, was anticipated by Scriven (1961). Difficulties similar to the problem of provisos have also been noted in ethics, e.g., with regard to Ross's (1930) definition of a "prima facie duty".)

One may be tempted to reject this problem by insisting that genuine law-statements lack provisos; on this view, that many familiar "lawstatements" are actually neither law-statements nor abbreviations for law-statements only goes to show that we have discovered very few genuine laws (or nomological explanations). To yield to this temptation would, I think, be unjustified. An account of laws must accommodate the fact that scientists show no reluctance to use these familiar claims in the manner distinctive of law-statements, e.g., in explanations and in support of counterfactuals. This fact would be difficult to explain if 
we held that scientists do not consider them to express laws. To insist nevertheless that only claims without provisos are genuine law-statements is to hold that whether a claim must or must not be captured by an analysis of what it is to believe a claim to state a physical law is not determined by whether scientists treat that claim as able or unable to perform those functions that distinguish law-statements from accidental generalizations. But, then, on what basis is the adequacy of an account of law to be evaluated? Scientific practice is the only phenomenon that exists for an account of law to save; if an account is tested against not actual science but science as idealized to conform to that account, the test is circular. If accounts of law are not free to disregard the fact that scientists treat "All gold cubes are smaller than one cubic mile" as an accidental generalization and "All cubes of Uranium-235 are smaller than one cubic mile" as expressing a law, even though this fact is troublesome for many proposed accounts, I see no reason why an account of law should be permitted to ignore the fact that scientists treat as law-statements many claims involving provisos.

Another temptation is to argue that although some law-statements involve provisos, these derive from other, more fundamental law-statements, which themselves need no provisos to describe regularities. For instance, the law of falling bodies follows in classical physics from the fundamental laws of motion and gravitation along with information about the earth; the proviso "... so long as the body is falling freely" restricts the law to those cases in which the gravitational-force law applied to this information about the earth accounts for all of the forces acting on the body. However, this temptation should also be resisted. It merely pushes the burden onto the fundamental laws: What regularity is described by the gravitational-force law? If it described a regularity between the masses and separation of two bodies and the total force that each exerts on the other, it would be false unless it included the proviso "... so long as the bodies exert no other forces upon each other." But to regard this proviso as part of this "fundamental" law not only conflicts with the law's applicability to a case in which two charged and massive bodies interact, but also raises the familiar problem: The proviso fails to specify the circumstances in which other forces are present, and so prevents the gravitational-force law from setting forth a particular relation. If each of the other force-laws is supposed to specify when a given non-gravitational force is present and thereby help to determine the relation asserted by the gravitational-force law, 
then each of these other force-laws would have to apply to a body affected by many types of forces and so could not describe a regularity involving the total force exerted on the body. Alternatively, for the gravitational-force law to describe a regularity between the masses and separation of two bodies and the gravitational (rather than total) force that each exerts on the other, a component gravitational force would have to be a real entity that conforms to certain regularities. That component forces are real is a controversial contention (see, e.g., Cartu'right 1983), and in any event, it seems to me that the nomic status of the gravitational-force law does not depend on it; after all, we use the Coriolis-force law in the manner distinctive of law-statements even though we believe there to be no Coriolis force to figure in a regularity that the law-statement might describe.

Perhaps one would be justified in setting the problem of provisos aside if one had some account of physical law that, except for this problem, were entirely successful. But since I know of no such account, I think it worth investigating whether greater progress toward one can be made by reflecting on provisos than by disregarding them. In this paper, I'll offer a response to the problem of provisos that ultimately undermines the regularity account of physical law and suggests an alternative, normative conception of law-statements according to which they specify the claims we ought to use, in various contexts, to justify certain other claims. I'll argue that some claims are properly adopted as law-statements although they are believed not to describe regularities, because one who believes that "It is $F$ " ought to be used to justify "It is $G$ " need not believe that some regularity, such as that all $F s$ are $G$, obtains.

In Section II, I'll explain the problem of provisos more fully, and in Section III, I'll attack Hempel's view of what a "law-statement" must be to qualify as complete, on which the problem depends. The more liberal criterion of completeness that I'll defend permits us to avoid Hempel's dilemma by enabling us to regard familiar "law-statements" as law-statements. I'll argue in section IV, however, that this response to the problem of provisos requires the rejection of any regularity account of physical law because many a claim commonly accepted as a law-statement describes no regularity; a normative conception of lawstatements then suggests itself. In Section V, I'll maintain that this strategy can be used to argue against many other conceptions of physical law besides the regularity account, such as those of Armstrong (1983) 
and Kneale (1949). Finally, I'll argue that my response to the problem of provisos is superior to that offered by Giere (1988).

Hempel does not explicitly present the problem of provisos as posing the dilemma that "law-statements" are either false or empty. Yet this dilemma certainly stands behind his discussion. It must be because he believes a "law-statement" without provisos would be false, and so would not be a law-statement, that Hempel defines provisos as "essential." He goes on to point out that if the complete law-statement includes the proviso $(x) \ldots$ if $P x$, then among the premises of an inference from the law-statement to testable predictions must be $P a$, as well as other auxiliary hypotheses. This might at first appear to reduce at least part of the problem of provisos to a special case of the DuhemQuine problem: a law-statement is not falsifiable (at least, not in a straightforward sense) because to make a prediction from it that can be tested, one must use auxiliary hypotheses, so one can preserve the law-statement, if the prediction fails, by rejecting an auxiliary hypothesis. If provisos are merely additional auxiliary hypotheses, distinguished from others only by the fact that they generally go unstated in scientific practice, then (it might appear) they do not represent a novel kind of threat to the falsifiability of individual hypotheses.

Hempel (1988, pp. 25f.) emphasizes, however, that the existence of provisos presents some obstacle to falsifiability beyond that posed by the Duhem-Quine problem. The Duhem-Quine problem assumes that the law-statements and auxiliary hypotheses are jointly sufficient to entail the testable prediction. But, Hempel maintains, if there are provisos among the auxiliary hypotheses, then this assumption often fails because, for many a familiar "law-statement," one can state neither the complete set of proviso conditions $(x) \ldots$ if $P x$ needed to make that "law-statement" true nor the complete set of auxiliary hypotheses $\mathrm{Pa}$ needed to infer the testable prediction from the law-statement. Therefore, Hempel says that in comparison to the Duhem-Quine problem, "[t]he argument from provisos leads rather to the stronger conclusion that even a comprehensive system of hypotheses or theoretical principles will not entail any [testable predictions] because the requisite deduction is subject to provisos" - that is to say, always remains subject 
to provisos, no matter how many auxiliary premises one adds to try to exclude all factors disturbing to the law.

Hence, it is only because he considers complete law-statements impossible, since "the number of provisos implicit in any law is indefinitely large" (Giere 1988, p. 40), that Hempel sees provisos as presenting a difficulty that is distinct from the Duhem-Quine problem. But suppose Hempel believed that a condition such as ". . . in the absence of other relevant factors" could appear in a law-statement, and likewise that "There are no other relevant factors" could function as an auxiliary hypothesis. Then he would have to admit that any law can be completely stated by a claim that includes only a finite number of conditions $(x) \ldots$ if $P x$, one of which might be "... if there are no disturbing factors," and that any inference to a testable prediction includes only a finite number of auxiliary hypotheses $P a$, one of which might be "There are no disturbing factors." The existence of provisos would then add nothing new to the Duhem-Quine problem. Hempel therefore must regard the expression "... in the absence of other relevant factors" as inappropriate for a law-statement. Though he does not explain why this is, the regularity account of laws, implicit throughout his discussion, suggests an answer: The sentence " $\Delta L=k \cdot L_{0} \cdot \Delta T$ obtains in the absence of factors that disturb it" states no definite relation and so cannot be a law-statement. This worry is evident in Giere's remark (1988, p. 40): “The problem is to formulate the needed restrictions without rendering the law completely trivial."

By Hempel's definition, a proviso $(x) \ldots$ if $P x$ usually is omitted from a statement of the law, and the corresponding premise $P a$ usually is not mentioned in inferences involving that law. Hempel regards these inferences as enthymemes and these familiar "law-statements" as incomplete. But why are we able to make do with incomplete lawstatements? And is Hempel correct in considering them incomplete? I'll now argue that Hempel's criterion of completeness is motivated by an incorrect view of what is necessary in order for a sentence to state a determinate relation. I'll argue that familiar law-statements, which include clauses such as "in the absence of other relevant factors," are complete as they stand.

That proviso premises are distinguished by their absence from ordin- 
ary conversation is not an incidental feature of Hempel's definition. As I've explained, it is bound up with the fact that the number of provisos is "indefinitely large," which makes it impossible to offer them all as premises. But why do scientists find it unnecessary to mention any of the proviso premises in order to put an end to demands for the justification of their conclusions? Why is it that although it is known that when someone is hammering on the bar, $\Delta L=k \cdot L_{0} \cdot \Delta T$ need not obtain, in actual practice a claim concerning $k, L_{0}$ and $\Delta T$ is recognized as sufficient, without "No one is hammering on the bar," to put an end to demands for the justification of a claim concerning $\Delta L$ ?

The answer is that in practice, when no one is hammering on the bar, nearly all of those who demand justifications of claims concerning $\Delta L$ already believe that this is so. (Likewise, to consider a different proviso example, it is widely understood by workers in many fields of physics that no cases will involve velocities approaching that of light.) Of course, to someone who presents a claim concerning $k, L_{0}$ and $\Delta T$ to justify a claim concerning $\Delta L$, one could object, "You have not told me that no one is hammering on the bar, and this you must do because if someone is, then (you will agree) your conclusion may well be false even though your premise is true." But apparently, that no one is hammering on the bar would, in nearly any actual case in which it is true, be believed in advance by those who might demand the justification of some claim concerning $\Delta L$. In nearly all cases, then, someone who demands a justification for a claim concerning $\Delta L$ should regard a claim concerning $k, L_{0}$ and $\Delta T$ as a sufficient response.

Attention to this kind of shared background not only explains why scientists needn't in practice give any of the "indefinitely large" number of proviso premises in order to justify their conclusions, but also reveals why Hempel's standard of completeness is too high. Hempel apparently considers a "law-statement" complete only if it suffices, in the absence of any background understanding, to inform one of what it takes for nature to obey the corresponding law. This ideal of completeness requires that the complete law-statement include all of the proviso conditions $(x) \ldots$ if $P x$ and, more importantly, that none of these conditions be "in the absence of disturbing factors," because this condition plainly appeals to background understanding. A generalization "All Fs are $G$, except when disturbing factors are present" does not indicate, in a manner intelligible to one who doesn't know already what constitutes a disturbing factor, some determinate regularity to which nature 
conforms. In exactly the same way, someone who is told to follow a rule "Conform to the regularity ... when it is appropriate to apply this rule" can understand what it would take to follow this rule only if she already knows when this rule is appropriately applied.

But to require that a rule be intelligible in the absence of implicit background understanding of how to apply it is not a reasonable criterion of completeness because no rule can satisfy it. As Wittgenstein (1958) suggests, one can always conceive of alternative interpretations of a rule that recognize the same actual past actions as conforming to the rule but regard different hypothetical actions as what it would take to follow the rule. It is futile to try to avoid this by including in the rule an expression that specifies explicitly how to apply the rest of the rule, for alternative interpretations of that expression are likewise conceivable. In the same way, a law-statement specifies a determinate relation only by exploiting implicit background understanding of what it would take for nature to obey this law. This point applies to any lawstatement, whether or not it blatantly appeals to implicit background understanding by referring to "disturbing factors."

That the proper way to apply a rule is not itself specified by any rule, intelligible without implicit background understanding of how to follow that rule, does not imply, as Hempel seems to think, that nothing counts as a violation of the given rule. The background understanding, albeit implicit, enables the rule to impose determinate requirements. This implicit understanding must be capable of being taught and of being made the explicit subject of discussion if disputes over it ever arise. Some fortune tellers explain away your failure to make accurate predictions by using their rules as the result of your having misapplied the claims they believe to be law-statements, of your having ignored some clause they say they neglected to mention. It is doubtful that they have undertaken any determinate commitments at all by adopting those "law-statements." It is as if someone says, "I can run a four-minute mile," but with each failure reveals a proviso that she had not stated earlier: "...except on this track," "... except on sunny Tuesdays in March," and so on. It quickly becomes apparent that this person will not acknowledge having committed herself to any claim by asserting "I can run a four-minute mile." Science is distinguished from such bunk neither by the explicit inclusion in scientific law-statements of all conditions Hempel would deem "essential" nor by the absence of implicit background understanding of how to apply those law-statements. What 
is noteworthy about science is that this background understanding is genuine background understanding. In general, all researchers identify the same testable claims as those to which one would become committed by adding a given lawlike hypothesis to a certain store of background beliefs. Because they agree on how to apply the hypothesis, it is subject to honest test.

\section{IV}

I'll now argue that this attractive response to the problem of provisos is incompatible with the view that law-statements describe regularities of a certain kind. This account of provisos leads instead to a conception of law-statements as specifying the claims we ought to respect, in a certain context, as able to justify certain other claims.

What, according to the regularity account, is the regularity stated by the familiar expression of the law of thermal expansion? Presumably, it is that a bar's length changes by $k \cdot L_{0} \cdot \Delta T$ whenever the bar has a certain composition, its initial length is $L_{0}$, its temperature changes by $\Delta T$, and there are no disturbing factors. That nearly all of us agree on whether "There are no disturbing factors" is appropriately said of a given case saves this expression, and so the law-statement, from emptiness; we share an implicit understanding of which predictions the lawstatement underwrites, of when it is properly applied. But whether certain scientists are correct in saying of a given case that there are no disturbing factors depends not just on the physical features of this case but also on their purposes, e.g., on the degree of approximation they can tolerate considering the use they intend to make of this prediction. Even if the regularity account can countenance as laws some uniformities involving the concerns of scientists, the law of thermal expansion was surely not supposed to be such a uniformity; somehow, the subject has changed from a law of physics to a law of the science of scientific activity.

It gets worse for the regularity account. Suppose that according to the regularity account, the familiar expression of the law of thermal expansion states that a bar's length changes by $k \cdot L_{0} \cdot \Delta T$ whenever the bar has a certain composition, its initial length is $L_{0}$, its temperature changes by $\Delta T$, no one is hammering on the bar hard enough to cause deviations from $\Delta L=k \cdot L_{0} \cdot \Delta T$ great enough (given our interests) to matter to us, and so on. Nevertheless, this claim cannot qualify as a 
law-statement according to the regularity account, for the relation it states does not obtain. It is violated, for example, when someone is hammering on the bar hard enough to cause the actual change in the bar's length to depart from $k \cdot L_{0} \cdot \Delta T$ but lightly enough for this departure to be irrelevant to the investigator's concerns. While in this case it is not true that the actual length of the bar changes by $k \cdot L_{0} \cdot \Delta T$, it is proper for such an investigator to predict that the length of the bar will change by $k \cdot L_{0} \cdot \Delta T$. The relation stated by the law involves not the bar's actual change in length but rather the change in length one is justified in predicting.

This suggests that the law of thermal expansion doesn't describe a regularity among events or states of affairs but concerns the way a claim concerning $\Delta L$ ought to be justified. The response I've advocated to the problem of provisos leads to a conception of the law of thermal expansion as the objective fact that under certain (partly pragmatic) circumstances, a premise about the bar's initial length, its change in temperature, and its composition ought to be used to justify a certain claim about its change in length. On this view, a law-statement has a normative element because it says that under certain circumstances, certain claims ought to be used to justify certain other claims.

To succeed, this account would have to show that law-statements are able to explain their instances, to support counterfactuals, and to be confirmed inductively by their instances because they specify the roles that certain descriptions should play in justifications. This account would likewise have to show that because accidental generalizations lack this prescriptive import, they cannot be used in these ways. To show this would be to break the familiar unilluminating circle of analysis from a law's explanatory power, to its physical necessity, to its capacity for counterfactual support, to its lawlikeness, to its capacity to be inductively confirmed by its instances, to its explanatory power. This task is well beyond the scope of this paper; I begin it in my (1993). My concern here is to show how this normative account of law-statements arises from a plausible response to the problem of provisos.

Let me summarize the argument. Contrary to Hempel, a law-statement that includes "so long as there are no disturbing factors" is not thereby rendered trivial. Like any other expression, this condition derives its content from an implicit shared understanding of how one should use it. Hence, the relation that a "law-statement" that includes this condition claims to obtain, which is determined by the appropriate 
way to apply this statement, ultimately depends on proprieties not codified explicitly. All there is to give meaning to "so long as there are no disturbing factors," and thereby save the "law-statement" from triviality, is how scientists consider the "law-statement" properly applied; because there is near unanimity on this point, the "law-statement" is not empty. But since the statement is properly applied to a given physical circumstance only when investigators have certain interests, the regularity that supposedly constitutes the law described by this statement must involve not only physical events but also investigators' concerns. Even if the regularity account regarded such uniformities as laws of physics, these uniformities do not obtain anyway and so, according to the regularity account, cannot be described by law-statements. For with regard to a given physical situation, it sometimes is and sometimes is not appropriate for us to say that $\Delta L$ will be $k \cdot L_{0} \cdot \Delta T$, depending on our concerns. But surely in a given physical situation, there is only one real amount by which the bar expands; the actual behavior of the bar does not depend on our interests. Once the lawstatement, in stating a relation involving "no one is hammering on the bar hard enough or otherwise disturbing it enough to matter to us," turns out to involve our interests, the other relatum, " $\Delta L$," is found to be infected by our interests as well. It refers to the change in the bar's length according to the claim that ought to be considered justified by these premises, which depends on the purpose for which we intend to use this claim, rather than to the bar's actual change in length, which does not. So the law-statement expresses a norm rather than a regularity involving the bar's real length. It states a relation between the presence of certain conditions (some having to do with our interests) and the way one ought to predict the bar's change in length.

A law-statement, then, informs an audience already able to tell whether there are "disturbing factors" that if there are none, they ought to use a given claim to justify another. On this view, a proviso is not "essential" tout court, contrary to Hempel. There is no fact of the matter to whether a law-statement has or lacks provisos. There is only whether those who would typically discuss that law-statement can learn, merely from reading it, what it prescribes they do (as is the case for the familiar expression of Newton's second law), or whether making the proviso explicit is essential for that audience.

Moreover, a normative conception of law-statements does not deny that there are regularities in nature. It denies only that law-statements, 
when performing their distinctive functions, are describing some of them. Laws, in the sense that the regularity account envisions them, need not be invoked to understand what law-statements say.

\section{V}

The foregoing argument, if successful, can be used to undermine not only any regularity account of law but also any account that takes a law-statement to describe a state of affairs that necessarily presupposes such a regularity. For instance, it has been suggested (e.g., by Kneale 1949) that law-statements describe not regularities of a certain kind but non-Humean connections of physical necessity. Such an account implies that the non-Humean connection supplements a regularity among circumstances. But with this regularity, the problem of provisos takes hold. The same reasoning can be deployed against recent accounts (see Armstrong, 1983, and Dretske, 1977) according to which lawstatements describe relations among universals, such as the property of lengthening by a given amount. On these accounts, the law-statement of thermal expansion entails that events conform to a certain relation "so long as there are no disturbing factors." Precisely what this comes to, i.e., whether some factor qualifies as "disturbing" or not, must be fixed by the law-statement. But, I have argued, the law-statement appeals to a determinate set of disturbing conditions only because it states a relation involving not the property of expanding in length by a given amount but the property of being able to serve as the subject of a justified claim attributing expansion by a given amount. In short, if the above reasoning goes through, it constitutes a recipe for an argument against any account according to which a law is or requires a regularity among events or states of affairs.

A law-statement concerning a particular influence, such as the Coriolis-force law or Newton's two-body gravitational-force law, suffices to tell persons having comparatively little background understanding how they ought to justify a certain claim. This is because the magnitude of the influence covered by the law-statement does not depend on which other influences are at work; hence, while there are provisos, there is none that demands significant background understanding, such as "in the absence of disturbing factors" would. The gravitational-force law, for example, specifies how one should justify a claim concerning the gravitational force between two bodies, whatever the other influences 
with which this subtotal should be combined to reach, say, the total force on a given body.

However, Giere's (1988) response to the problem of provisos does not capture the fact that to be committed to the gravitational-force law is to be committed to treating a certain inference to the component gravitational force as correct, no matter what the other relevant influences. In light of the problem of provisos, Giere holds certain familiar law-statements to be false as claims about the physical world, but he contends that, as law-statements, they must nevertheless function as descriptions of something. He therefore tries to find something that they describe. While this search has led Armstrong and Dretske to the exotic realm of universals, Giere (along with Cartwright, 1983, whose account is similar in all relevant respects) maintains that a law-statement describes a scientific model. One may hold that the relevant behavior of a given real system can be predicted by using some model; Giere terms such a claim a "theoretical hypothesis." Since the law-statement describes the model, not reality, it needs no qualification by provisos to be accurate.

Consider, then, what Giere says (1988, p. 44) about two laws, each concerning a single influence, that are combined to account for a magnetically influenced pendulum:

We have discovered a new kind of pendulum ... in which the force of gravity is supplemented by a magnetic force directed toward a point below the point of rest. Constructing a theoretical model that does apply to such systems is a fairly easy problem in physics.

If the gravitational-force law specifies how one ought to calculate a subtotal (the gravitational influence of one body on another) no matter what the other influences present, and the magnetic-force law does likewise, then this is indeed an easy problem. Having already accepted these law-statements, and having recognized the proper way to add forces and to use the net force on a body to infer its acceleration, we are committed to a particular procedure for predicting the bob's motion.

But Giere takes these law-statements not to prescribe which models to use (which is the job of theoretical hypotheses) but merely to describe certain models. Thus, Giere must admit that by accepting that the gravitational-force law describes certain models, we are not committed to saying that one ought to use a model it describes to predict the bob's motion. Moreover, one who accepts the theoretical hypothesis that this law should be used to calculate the gravitational force exerted by the 
earth on a non-magnetic bob is not thereby committed to the theoretical hypothesis that this law should be used to calculate the gravitational force exerted by the earth on a magnetically influenced bob.

On my view, in contrast, to accept this law-statement is to recognize the way one should justify claims concerning the component gravitational force, whatever the other relevant influences. This view accounts for what Giere's view obscures: That we are committed to some common element in our treatments of ordinary and magnetically augmented pendula (namely, an identical way of justifying a certain subtotal) in virtue of which the magnetically augmented pendulum is an easy problem. We became committed to elements of its solution when we adopted solutions to other problems.

I have argued against Hempel's contention that a complete lawstatement must specify its own range without depending on implicit background understanding in order to do so. I have also argued against Giere's (and Cartwright's) alternative claim that a law-statement says nothing about its range, leaving it for theoretical hypotheses to specify. I have defended the view that a law-statement specifies its range in a fashion that may involve blatant appeal to implicit proprieties of use. I have thereby tried to offer a way around the problem of provisos, at the price of abandoning the regularity account of law in favor of a normative analysis. Further discussion of that proposal must await another occasion.

\section{REFERENCES}

Armstrong, D.: 1983, What Is a Law of Nature?, Cambridge University Press, Cambridge. Ayer, A. J.: 1963, "What is a Law of Nature?", in The Concept of a Person and Other Essays, Macmillan, London, pp. 209-234.

Braithwaite, R. B.: 1953, Scientific Explanation, Cambridge University Press, Cambridge.

$\rightarrow$ Canfield, J. and Lehrer, K.: 1961, 'A Note on Prediction and Deduction', Philosophy of Science 28, 204-208.

Cartwright, N.: 1983, How the Laws of Physics Lie, Clarendon Press, Oxford.

Coffa, J.: 1968, 'Discussion: Deductive Predictions', Philosophy of Science 35, 279-283.

$\rightarrow$ Dretske, F.: 1977, 'Laws of Nature', Philosophy of Science 44, 248-268.

Giere, R.: 1988, 'Laws, Theories, and Generalizations', in A. Grunbaum and W. Salmon (eds.), The Limits of Deductivism, University of California Press, Berkeley, pp. 3746.

Goodman, N.: 1983, Fact, Fiction, and Forecast, 4th ed., Harvard University Press, Cambridge. 
Hempel, C. G.: 1965a, 'Studies in the Logic of Explanation', in Aspects of Scientific Explanation, The Free Press, New York, pp. 245-295.

Hempel, C. G.: 1965b, 'Typological Methods in the Natural and the Social Sciences', in Ibid., pp. 155-172.

Hempel, C. G.: 1988, 'Provisos', in A. Grunbaum and W. Salmon (eds.), The Limits of Deductivism, University of California Press, Berkeley, pp. 19-36.

Kneale, W.: 1949, Probabilitv and Induction, Oxford University Press, Oxford.

Lange, M. B.: 1993, 'Lawlikeness', Noûs, forthcoming.

Lewis, D. K.: 1973, Counterfactuals, Harvard University Press. Cambridge.

Lewis, D. K.: 1986, 'Postscript to 'A Subjectivist's Guide to Objective Chance", in Philosophical Papers: Volume 2, Oxford University Press. Oxford, pp. 121-126.

Mackie, J. L.: 1962, 'Counterfactuals and Causal Laws'. in R. S. Butler (ed.). Analytic Philosophy, Barnes and Noble, New York, pp. 66-80.

Nagel, E.: 1961, The Structure of Science, Harcourt, Brace, and World, New York.

Reichenbach, H.: 1947, Elements of Symbolic Logic, Macmillan, New York.

Ross, W. D.: 1930, The Right and The Good, Oxford University Press, Oxford.

Scriven, M.: 1961, 'The Key Property of Physical Laws - Inaccuracy', in H. Feigl and G. Maxwell (eds.), Current Issues in the Philosophy of Science, Holt, Rinehart, and Winston, New York, pp. 91-104.

Wittgenstein, L.: 1958, Philosophical Investigations, 3rd ed., trans. G. E. M. Anscombe, Macmillan, New York.

Manuscript submitted September 26, 1991

Final version received April 21, 1992

Department of Philosophy

University of California, Los Angeles

405 Hilgard Avenue

Los Angeles, California 90024-1451

U.S.A. 